\title{
Heterogeneous network optimization using robust power-and-resource based algorithm
}

\author{
Savita Patil ${ }^{1}$, A.M. Bhavikatti ${ }^{2}$ \\ ${ }^{1}$ AMC Engineering College, India \\ ${ }^{2}$ Computer Science and Engineering, Bheemanna Khandre Institute of Technology (BKIT), India
}

\begin{abstract}
Article Info
Article history:

Received Sep 17, 2018

Revised Mar 13, 2019

Accepted Apr 24, 2019

Keywords:

Base stations (BS)

Heterogeneous networks

(HetNets)

Robust power-and-resource

based algorithm (RPRA)

ABSTRACT

In order to meet the increasing mobile data-traffic, spatial densification of network with several low-power nodes, the high-power macro BS and HetNet are the major key enabling solution. However, the HetNet is unplanned in nature, causes irregularities and interferences that without any user association rules. The appropriate deployment of the femto-cell in HetNet can provide effective traffic offloading, where the alleviate mobbing in the macro-cells can decrease the power consumption therefore it optimizes the user experience. Moreover, the protection is also important for the macro and femto cell users in a network through maintaining the min-max level of interferences. In this paper, we proposed RPRA that comprises two robust approach such as robust power-controller and the robust channel-allocation approach, which can improve the spectral efficiency and user experiences at lower network coverage areas via eliminating the week coverage zones. Also provide high user rate connection by effective interference in an efficient spectrum, lowering in transmission power and cost-effectiveness via less time delay. To show the effectiveness of our proposed model we have compared with several existing techniques and we got significant improvement in throughput, also reduction in time delay and transmission power.
\end{abstract}

Copyright () 2019 Institute of Advanced Engineering and Science. All rights reserved.

\section{Corresponding Author:}

Savita Patil,

AMC Engineering College,

18th K.M, Bannerghatta Main Rd, Bengaluru, Karnataka 560083, India.

Email: sampati1949@gmail.com

\section{INTRODUCTION}

The present cellular network faces the problem of huge increment of data traffic, in that the spectral efficiency allocation and design is very important. In general, the concept of frequency reuse is the major key for 4-Generation and the future cellular networks; however, the HetNets have attracted lot of attention of the researchers in LTE (Long-Term-Evolution) wireless network [1]. Moreover, the potential of HetNets has used to optimize the spectral efficiency and user experiences at very dense environment 'or' lower network coverage areas, though the deployment of HetNets to small-coverage area (femto 'or' Pico) and low power cell with in the macro-cell environment is generally eliminate the week coverage zones, also provide the higher "spectral-efficiency" [2]. The appropriate deployment of the "femto-cell" in HetNet can provide effective traffic offloading, where the alleviate mobbing in the macro-cells can decrease the power consumption therefore it optimizes the user experience. In order to develop macro-femto based HetNets, the main concern is to manage the co-channel interferences that occurs because of spectrum sharing, the interferences of co-channel may become worse due to femto BSs that installed through end-users without any of centralized planning procedure. Generally, femto BSs are deployed under macro-cell areas without any warning due to special requirement of client, therefore the lack centralized-cell planning the effect of interferences in two-tier "macro-femto HetNets" vary considerably in a conventional cell networks. 
A main difficulty occurs in deployment process of HetNet in order to provide high user rate connection by effective interference in a spectrum efficient and cost-effective manner, several published works has focused on "co-channel interference" difficulty that arises between femto BSs and macro BSs [1]. Through studding several works, the demonstration femto BS as the cognitive radios permits femto BS to sense macro BS user performance to control the type of interference at the available spectral resources; however, the problem related to power controller has discussed, where the network configuration is femtomacro two-tier networks through proposing downlink based power allocation algorithm, which consist information of channel for all involved links in network. While considering a centralized based femto gateway process that called as fusion center, is basically relies on gateway connection in order to distribute and collect the necessary channel interference information. An extensive amount of works has been done with respect to user association in the HetNets considering different type of scenarios, like as in [3] has proposed a joint optimization procedure of user association such as channel selection and transmission power has considered to minimalize the as per the user throughput. While in [4], the author proposed for the adaptive association rule in order to enlarge the consisting number of users through decreasing the total usage cost of radio resources under a HetNet environment with relay nodes; whereas in [5] they used integrated user association and resource allocation in order to increase the metric of proportional fair (i.e., log-rate sum).

The decentralized based interference management protocol for the cognitive femto BSs, where each femto BSs will allocate its spectrum through considering the potential interference and interference from the neighborhood femto-cells is discussed in [6]. The methodology necessary for communication based interference channel are very effective among the neighborhood channel, though the direct link in between macro BS and femto BS are very important in an channel interference, in [7] they discussed about the resources allocation optimization process. In [8], they valuate the channel allocation strategy through applying the game-theory approach for cognitive femto BS radio management resources; furthermore, in [9] they studied the distribution approach for the self-originated cell network in order to control the transmit powers on several different channels that to maintain user quality and the channel conditions. A threshold based spectrum sensing is very difficult to design, where in order to get the high desired trade-off in between outage probability and spatial frequency a parameter need to be tuned carefully, the more lesser the threshold of spectrum sensing the lower the aggregate interference and larger the spectrum sensing range. However, the reuse of same frequency channel can be done after the larger 'spatial intervals' that tends in low spatial frequency reuse, the analysis and designing of this type of trade-off in two-tier HetNet with femto-cells is discussed in [10].

However, the main aim of power controller is to decrease the transmission power that tends to decrease the high power consumption, also reduces the interference at inter-cell that is very important as per the above discussion; therefore, the altering the power transmission with respect to resource block is essential to achieve high bit-rate in femto-cell and pico-cell, where the occurred interference can be reduce significantly in small-cell network. Therefore, the channel capacity and coverage of poor area under a HetNet scenario is important for enhanced user services. Moreover, the protection is also important for the macro and femto cell users in a network through maintaining the min-max level of interferences, the QoS effectiveness under a traffic is major factor because this can damage the resource allocation procedure that tends to degrade the QoS of users in a small network (i.e., Pico or femto cells). In general, the increment of number of mobile users can cause the decrement in QoS due to more bandwidth requirement, in order to handle this uncertainty, the several femto BSs are required, therefore the proposed algorithm should be robust in nature so that it can handle uncertainties effectively. In this paper, we proposed RPRA that comprises two robust approach such as Robustified Power Controller (RPC) and the Robust Channel-resource Allocation Approach (RCAA), which can improve the spectral efficiency and user experiences at lower network coverage areas via eliminating the week coverage zones. Also provide high user rate connection by effective interference in an efficient spectrum, lowering in transmission power and cost-effectiveness via less time delay. The implementation of power controller used real time based scenario (i.e., HeNB) for macro-cell and femtocell, also the standard dynamic representation is performed for power controller [11], which is effective at power switching points in HetNets. In result analysis we shown the performance of our proposed model with respect to existing algorithm under different considered scenarios to validate the model performance.

\section{LITERATURE SURVEY}

The major challenge is to enhance the indoor area coverage, the study in [12] shows more than 70\% of the traffic data and $50 \%$ of all voice calls are make from indoors environment. So the coverage of indoor environment can provide higher data-rate and QoS, which are the major problem while developing the upcoming generation (Wireless Communication Networks) WSNs, however providing macro-cell BS in order to handle the growing traffic demand is much expensive. Therefore, a novel future generation architecture is 
suggested to overcome the ongoing demand of data traffic and it consist of femto-cell BSs (FBS) [13, 14]. The presence of proximity at receiver and transmitter causes the better users experience and able to communicate optimize throughput, suppose the indoor user such as ones in their homes or apartments are linked to an FBS, and some of indoor users communicate directly through the macro-cell that tends to optimize the performance of network. Considering the operators side, the femto-cell can increase the reuse of spectrum in order to provide the high spectral efficiency and the network capacity, moreover, the FBS are compensated and upheld via the owners. Inspire of these advantages it comes with several new challenges such as in synchronization, interference management and network architecture [14].

In general, the difficulty due interferences have become major problem which requires novel solution because of more complexity in comparison with the existing standard cellular network. Basically there are several type of deployment architecture is present for femto-cell networks. In one of the type of orthogonal deployment, the spectrum is sliced into dual independent fragments, where one is used via the femtocells and other is used by the macro-cells [15], moreover, they remove inter-tier level interferences due to that resources of frequency are not fully utilized. In other type of channel deployment architecture, the both femto and micro cells having directly access to available channels [16], this type of methodology can generate large interference levels. However, there is also partial 'co-channel' deployment approach, such that the all presented spectrums are separated into two parts; one specially dedicated to macro-cells and the second one shared to femto and macro cells $[17,18]$. These deployment model of FBS access approach is used to restrict 'or' allow the usage of spectrum by users. However, the open access provide user to have access, while the closed access only allows subscribed users for spectrum access (i.e., closed subscriber group), moreover the use of hybrid access allows also to non-subscriber to use particular amount of the 'femto-cell' resources. In paper [19], they multi casting type of scheduling problem in HetNets via a "half-duplex relay" station is discussed and aim is to minimalize the transmission delay of block (i.e., packets to users) over the time period via rate-less codes. Therefore, the operation of half-duplex is performed at each time interval, relay station is select a multicast packet to users 'or' realize a packet from macro BS, afterwards they formulated the fluid relaxation to optimize the decision difficulty and provide the optimal approach for threshold base deployment in order to exploit the multicast speculation channel.

Therefore, the relay station only multi-cast whenever the channel capacity has reached considerably high, then an online policy-based relaxation that does not needed the information of channel distribution. The channel distribution is considered to be symmetric to all users and the closed form of asymptotic expression is performed by their policy. While, considering dual user model, they used an asymptotically optimal function that based on the "water filling" rate allocation for approximating the better model policy. The user scheduling ('US') and user association ('UA') has consider for the load balancing at downlink operation of wireless HetNet via formulating the maximum utilization of wide network problem to resolve the efficiency problem [20]. First of all, they approximate the throughput of non-convex problem with US in order to concave the functionality and this can demonstrate the difference of approximation algorithm zero whenever the present users are much larger. Afterwards, distributed convex approach that is known for change the multipliers direction methodology is used as the integrated US-UA algorithm that can be applied on BS and user sides individually and, proposed to be resource allocation and BS association solutions. The proposed model feature enhancement can be used in future to improve performance that apart from multi-user diversity and load balancing.

The trade-off in between the energy consumption and data-rate performance in non-orthogonal multiple access ('NOMA') has studied in [21], where the problem of scheduling and the power enhancement in NOMA HetNets is performed; though the power allocation and energy efficient scheduling approach in NOMA HetNets are introduced for both imperfect and perfect CSI. The FQL (Fuzzy-Q-Learning) based energy efficient approach (i.e., include either sleep 'or' wake-up model) for the BSs in a HetNet and the aim is to minimalize the energy without effecting the offered QoS through performing switch off operation at redundant BSs in accordance to native traffic, which depend upon the coverage area of cell. The sleep-mode activity for BSs may tends to a large coverage loss, unless some particular optimize solution is occurred at same interval [22]. The HetNets consist of secondary and primary user, whereas the ordinary cellular users are considering to be primary users (PUs), while unlicensed, IoT and some sensor equipment are considering to be secondary users (SUs) in [23]. The channels HetNets are occupied PUs while the SUs can only reuse the PUs channels, here they considered dual mode transmission for the SUs such as SU only come up with BS directly 'or' via the help of associative relay node and the energy efficiency enhancement of SUs prototype is considered.

In general, they majorly aim on association of users such as selection of BS, mode selection and channel allocation in order to optimize the power control at uplink communication in between the BSs and $\mathrm{SU}$; moreover, these type of difficulty can be formulated as mixed integer optimize and non-convex problem. An opportunistic type of power controller approach is proposed in [24] to mitigate the interference from the 
active number of femtocells at uplink transmission, the macro-cell BS chooses the allowance interference as per number of femto-cell users and femto-cells, afterwards allocate the transmit power under cross-tier interference in considered HetNets. The approach towards the power controller should verify the active number of femtocells as per the effective controller, also they proposed two kind of sensing approaches to predict the active femtocells such as; centralized and the distributed, respectively. The difficult outage probability function under the Rayleigh fading effect has studied in [25] and solve a convergence problem for initially discovered approaches in the case of interference. Afterwards, they address the power related difficulty with holding the outage constraints also considering their feasible environment; moreover, a dynamic approach has proposed to provide outage probability condition in a HetNet in order to decrease the overall energy consumption, also this guarantee to all femto-cell users a bound of min and max for the worst level of outage probability.

\section{PROPOSED METHODOLOGY}

In general, the interferences have become major problem which requires novel solution because of more complexity in comparison with the existing standard cellular network. A new type of deployment architecture is present such as femto-cell networks. In one of the type of orthogonal deployment, the spectrum is sliced into dual independent fragments, where one is used via the femtocells and other is used by the macro-cells. In this study, we consider one macro-cell and several number of femto-cells, where femto-cells has able to decrease the interference level with various channel signals that will help to optimize the spectral efficiency as per the covered cell area. The several number of femto-cells can be located in a macro-cell, therefore the BSs of femto-cells are located at very shorted distance to each other, which help to cover the poor transmission area present in a macro-cell; moreover, the equipment becomes more energy efficient due to the shorted distance via decrement in transmission power that help to increase the life of battery. In general, femto-cells are used in indoor location so that the macro-cell can provide the robust services to users due to its reduction of overhead. Block diagram of proposed RPRA is shown in Figure 1, where the proposed approach shows consider robust approach that can handle variation in BSs (i.e., different scenarios), which will be shown in result analysis section.

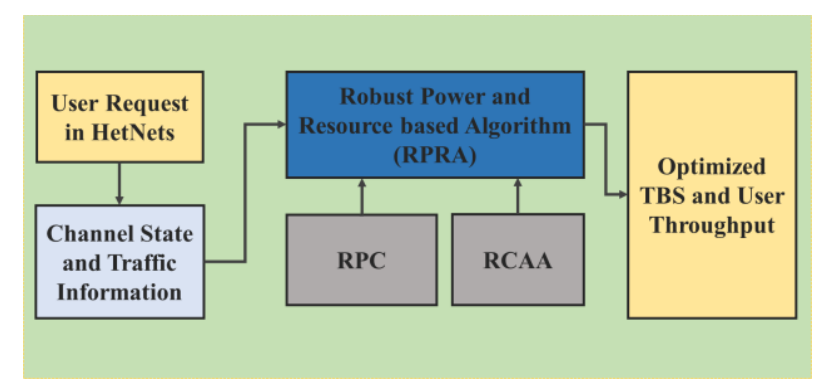

Figure 1. Block diagram of proposed RPRA

In this section, first we will provide the detailed study and numerical formulation of Robustified Power Controller (RPC) and afterwards we will go for the Robust Channel Allocation Approach (RCAA). In consider HetNet, the users of femto-cells and macro-cells makes the request, based upon their requirement the channel and traffic details are acquired, then all these things are forwarded to resource allocation and power control process for further optimization process, so we can get maximum user throughput and optimize power transmission.

\subsection{Robustified power controller}

Considering the channel uncertainties, the femto-cell user (FCU) requirements and macro-cell user (MCU) performance protection is very necessary to considered simultaneously, therefore we consider the robust power controller and resource allocation approach in a two-tier HetNet under the 'stochastic model'. A multi-user OFDM based HetNets is considered that consist $D$ number of FCUs, communicating with associated femto-cell BSs over the $E$ number of subcarrier, moreover FCUs are used to operate the MCUs via FC-BSs, where $D$ and $E$ are changes according to the number of user's active and available subcarrier channel, that can be given as $\in \in \mathfrak{D} \triangleq\{1,2,3 \ldots \ldots, D\}$ and $e \in \mathcal{E} \triangleq\{1,2,3 \ldots \ldots, E\}$, where, we assumed that $\mathcal{E} \geq \mathfrak{D}$ and the subcarrier bandwidth is expected to be $F \mathrm{~Hz}$ which is much less in comparative to the 
wireless channel bandwidth. Therefore, applying Shannon Hartley Theorem (SHT) [26] corresponding FCU data rate $d$ at subcarrier $e$ is written as.

$$
g_{d, e}=F h_{d, e} \log _{2}\left(1+I_{d, e} J_{d, e} / K_{d, e}\right)
$$

Where, $h_{d, e}$ shows the $d$ FCU subcarrier task at the $e$ subcarrier, $K_{d, e}$ shows the $d$ FCU background noise at subcarrier $e, J_{d, e}$ shows the $d$ FCU straight channel gain at subcarrier $e$ and, $I_{d, e}$ shows $d$ FCU transmit power at subcarrier $e$. There is 0 'or' 1 subcarrier assignment which signify the subcarrier $e$ used via $d$ FCU or not. The main problem with this is battery capacity at the $m$ th FCU transmitter, therefore the constraint can be written as:

$$
\sum_{e=1}^{E} h_{d, e} I_{d, e} \leq I_{d}^{\max }, \forall d \in \mathfrak{D}
$$

In above equation, $I_{d}^{\max }$ shows the maximal power transmit of the FCU, where the data-rate should accomplish the nominal requirement of $d$ FCU QoS so that it can be written as:

$$
\sum_{e=1}^{E} g_{d, e} \geq G_{d}^{\min }, \forall d \in \mathfrak{D}
$$

Where, $G_{d}^{\min }$ shows the minimum requirement-rate of $d \mathrm{FCU}$, also interference constraint of the total cross-tier under femtocell HetNet to the MCU receiver section can be given as:

$$
\sum_{d=1}^{D} \sum_{e=1}^{E} h_{d, e} I_{d, e} N_{d, e} \leq M^{i l}
$$

The interference level at receiver side of MCU is denote by $M^{i l}$, the sum rate maximization by power controller at the HetNets scenario can be written as:

$$
\begin{aligned}
& \max \\
& h_{d, e} I_{d, e} \sum_{d=1}^{D} \sum_{e=1}^{E} g_{d, e} \\
& \sum_{e=1}^{E} h_{d, e}=1, \forall d \in \mathfrak{D}, Z_{1} \\
& \sum_{k=1}^{K} h_{d, e} I_{d, e} \leq I_{d}^{\max }, \forall d \in \mathfrak{D}, Z_{2}
\end{aligned}
$$

Where, $Z_{1}$ denotes the individual subcarrier $e$ that is allocated to each $\mathrm{FCU}, I_{d, e}=1$ shows the $e$ thsubcarrier which is used via $d \mathrm{FCU}$, and $Z_{2}$ is used to show the constraint of power transmission at $d$ FCU over the sub-carrier.

$$
\begin{aligned}
& \sum_{e=1}^{E} G_{d, e} \geq G_{d}^{\min }, \forall d \in \mathfrak{D}, Z_{3} \\
& \sum_{\mathrm{d}=1}^{\mathrm{D}} \sum_{\mathrm{e}=1}^{\mathrm{E}} \mathrm{h}_{\mathrm{d}, \mathrm{e}} \mathrm{I}_{\mathrm{d}, \mathrm{e}} \mathrm{N}_{\mathrm{d}, \mathrm{e}} \leq \mathrm{M}^{\mathrm{il}}, \mathrm{Z}_{4} \\
& h_{d, e} \in\{0,1\}, \forall d \in \mathfrak{D}, e \in \mathcal{E}, Z_{5}
\end{aligned}
$$

The (6) confirm the QoS for each FCUs, where, $Z_{4}$ denotes the interference of total power at MCU receiver, and the main problem is $h_{d, e}=1$ which is mixed integer and "non-convex programming" difficulty, $N_{d, e}$ signifies the feedback of channel gains that provided through MCU to FCU. In recent development, majority of the researchers has aimed towards power allocation strategy in the HetNets [27] which focus on improvement power with perfect CSI [28]. However, in particle scenario, the quantization errors presence reasons the channel uncertainty which is harmful for MCUs, in order to overcome this problem, we should consider some improve technique so that can deal with these type of uncertainties. Therefore, we use Robustified power controller and, the (6) can be rewritten in a probability form:

$$
\begin{aligned}
& \max _{h_{d, e} I_{d, e}} \sum_{d=1}^{D} \sum_{e=1}^{E} g_{d, e} \text { s.t. } Z_{1}, Z_{2}, Z_{5} \\
& \mathrm{P}\left\{\sum_{e=1}^{E} g_{d, e} \leq G_{d}^{\min }\right\} \leq Q_{d}, \forall d \in \mathfrak{D}, Z_{6} \\
& \mathrm{P}\left\{\sum_{d=1}^{D} \sum_{e=1}^{E} h_{d, e} I_{d, e} N_{d, e}>M^{i l}\right\} \leq \mathfrak{z}
\end{aligned}
$$

Where, both (7) guarantee the QoS of MCU and FCU through using probability function, 3 and $Q_{d}$ shows for the threshold value used in outage probability. OFDM feature method has consider, therefore the subcarriers are independent from each sub-carrier, though each of FCU information are mutually independent from subcarriers and the data-rate set is defined as: 


$$
\begin{aligned}
& S^{e}=\left\{g_{d, e} \leq G_{d}^{\text {min }}\right\} \\
& S=\left\{\sum_{e=1}^{E} g_{d, e} \leq G_{d}^{\text {min }}\right\}
\end{aligned}
$$

Where, $S$ set is an intersection subset of $S^{e}$ such as:

$$
\bar{S} \subset S=S^{1} \cap S^{2} \ldots S^{e}
$$

Applying the probability analysis in (9), we got the following relationship:

$$
\{\bar{S}\} \leq \mathrm{P}\{S\}=\prod_{e=1}^{E} \mathrm{P}\left\{S^{e}\right\}
$$

Furthermore, it can be given as:

$$
\mathrm{P}\left\{\sum_{e=1}^{E} g_{d, e} \leq G_{d}^{\min }\right\} \leq \prod_{e=1}^{E} \mathrm{P}\left\{g_{d, e} \leq G_{d}^{\min }\right\}
$$

Probabilistic constraint rate for the upper bound should fulfils the essential outage probability throughout the worst scenario, therefore the condition scenario by (7) can be written as:

$$
\operatorname{Max} \mathrm{P}\left\{\sum_{e=1}^{E} g_{d, e} \leq G_{d}^{\min }\right\} \leq \prod_{e=1}^{E} \mathrm{P}\left\{g_{d, e} \leq G_{d}^{\min }\right\} \leq Q_{d}
$$

The deterministic outage probability of (12) can be given as:

$$
G_{d}^{\min } \leq F h_{d, e} \log _{2}\left(1+\frac{I_{d, e}}{K_{d, e}} \mathrm{~J}_{J_{d, e}}^{-1}\left(Q_{d} / E\right)\right), \forall d \in \mathfrak{D}
$$

The (13) confirm the 'power transmission' with the outage probability, so the probabilistic interference from (7) can be updated as:

$$
\mathrm{h}_{\mathrm{d}, \mathrm{e}} \mathrm{I}_{\mathrm{d}, \mathrm{e}} \leq \frac{\mathrm{M}^{\mathrm{il}}}{\operatorname{EN}_{\mathrm{N}_{\mathrm{d}, \mathrm{e}}}^{-1}(\sqrt[\mathrm{DE}]{1-3})}, \forall \mathrm{d} \in \mathfrak{D}, \forall \mathrm{e} \in \mathcal{E}
$$

Therefore, the (14) is said to be deterministic in nature and it is necessitating to keep it as presentable form, moreover, the difficulty of power controller without any prior information can be given as:

$$
\begin{aligned}
& \max _{h_{d, e} I_{d, e}} \sum_{d=1}^{D} \sum_{e=1}^{E} g_{d, e} \text { s.t. } Z_{1}, Z_{2}, Z_{5} \\
& F h_{d, e} \log _{2}\left(1+\frac{h_{d, e}}{K_{d, e}} \mathrm{~J}_{J_{d, e}}^{-1}\left(Q_{d} / E\right)\right) \geq G_{d}^{\text {min }}, d \in \mathfrak{D} .
\end{aligned}
$$

Here, the inverse function of collective distribution at variable $J_{d, e}$ and $N_{d, e}$ is applied and can be written

$$
\begin{aligned}
& \text { as } \mathrm{J}_{\mathrm{J}_{\mathrm{d}, \mathrm{e}}}^{-1} \text { and } \mathrm{N}_{\mathrm{N}_{\mathrm{d}, \mathrm{e}}}^{-1} \\
& \operatorname{Eh}_{\mathrm{d}, \mathrm{e}} \mathrm{I}_{\mathrm{d}, \mathrm{e}} \mathrm{N}_{\mathrm{N}_{\mathrm{d}, \mathrm{e}}}^{-1}(\sqrt[\mathrm{DE}]{1-3}) \leq \mathrm{M}^{\mathrm{il}}
\end{aligned}
$$

In general, the FCUs can obtain the CSI by the channel estimation procedure between the FCUs and the MCUs, therefore these can arise some trouble at CSI acquisition. Therefore, we consider the self-determining prototype of Gaussian distribution in order to handle the ambiguity parameters. However, the FCUs transmitter channel gain to BS is obtain by a robust user-quantizer afterwards the feedback is send back to the corresponding FCUs transmitter.

\subsection{Robust resource allocation approach}

The major difficulty is still robustification at channel allocation process due the $h_{d, e}$ integer variable, the both integer and real time variable such as $I_{d, e}$ and $h_{d, e}$ are under optimization problem, therefore it conducts diverse integer programming difficulty, so the relaxation of subcarrier assignment feature into the continuous one that can be also shown as variable $p_{d, e}=I_{d, e} h_{d, e}$ for a femto user and subcarrier. Where, $h_{d, e} \in[0,1]$ signifies a time division strategy for the various femto users. Therefore, in order to obtain the $p_{d, e}$ and $h_{d, e}$ condition variables we apply Karush-Kuhn-Tucker (KKT) [29], which is 
nonlinear programming approach that allows equality constraints variables and based on the Lagrange multipliers method, thus the derivative form of optimal variables can be written as:

$$
\begin{aligned}
& 0 \leq p_{d, e} \perp \frac{\partial R_{d, e}(\ldots)}{\partial p_{d, e}} \\
& 0 \leq h_{d, e} \perp \frac{\partial R_{d, e}(\ldots)}{\partial h_{d, e}}
\end{aligned}
$$

Where, $\perp$ shows the orthogonal relation to corresponding variables and $L_{m, k}$ shows the Lagrange function, in addition, the $\frac{\partial R_{d, e}(\ldots)}{\partial p_{d, e}}$ and $\frac{\partial R_{d, e}(\ldots)}{\partial h_{d, e}}$ derivatives can be written as:

$$
\begin{aligned}
& \frac{\partial R_{d, e}(\ldots)}{\partial h_{d, e}}=\left(1+\mu_{d}\right) \frac{\partial \tilde{g}_{d, e}\left(p_{d, e}, h_{d, e}\right)}{\partial p_{d, e}}-\lambda_{d}-u\left(\widehat{N}_{d, e}+Q^{-1}(1-\jmath) u_{d, e}\right) \\
& \frac{\partial R_{d, e}(\ldots)}{\partial h_{d, e}}=\left(1+\mu_{d}\right) \frac{\partial \tilde{g}_{d, e}\left(p_{d^{*}, e}, h_{d, e}\right)}{\partial h_{d, e}}-\beta_{d}
\end{aligned}
$$

Where, $\mu_{d}, \beta_{d}, u$ and $\lambda_{d}$ are nonnegative Lagrangian multipliers for corresponding constraints, inverse Gaussian Q-function is given by $Q^{-1}($.$) . Therefore, the optimal transmit power with the channel allocation$ can be written as:

$$
I_{d, e}^{*}=\frac{p_{d, e}}{h_{d, e}}=\left[\frac{1}{\ln 2} \frac{F\left(1+\mu_{d}\right)}{\lambda_{d}+u\left(\widehat{N}_{d, e}+Q^{-1}(1-3) u_{d, e}\right)}-\frac{K_{d, e}}{\hat{J}_{d, e}+2 \mathfrak{w}_{d, e} \mathfrak{s}_{d}}\right]^{+}
$$

Where, outage probability threshold is $\mathfrak{s}_{d}$ and the upper bound region is $\mathfrak{w}_{d, e},[\mathfrak{x}]^{+}=\max (0, \mathfrak{x})$ and the subcarrier $e$ is allocated to the $d^{*}$ optimal user such as:

$$
h_{d, e}^{*}=\left.1\right|_{d^{*}=\max _{d} \widehat{\beta}_{d, e}}
$$

Where, $\hat{\beta}_{d, e}$ is given by:

$$
\hat{\beta}_{d, e}=\left(1+\mu_{d}\right) \frac{\partial \tilde{g}_{d, e}\left(p_{d^{*}, e}, h_{d, e}\right)}{\partial h_{d, e}}=F\left(1+\mu_{d}\right)\left(\log _{2}\left(1+\frac{\hat{J}_{d, e} I_{d, e}^{*}}{K_{d, e}}\right)-\frac{\hat{J}_{d, e} I_{d, e}^{*}}{\ln 2\left(K_{d, e}+\hat{J}_{d, e} I_{d, e}^{*}\right)}\right)
$$

Therefore, the Lagrangian multipliers is upgraded as:

$$
\begin{aligned}
& \lambda_{d}^{t+1}=\left[\lambda_{d}^{t}-x_{1}^{t}\left(I_{d}^{\max }-\sum_{e \in Z_{d}} I_{d, e}^{t}\right)\right]^{+} \\
& \mu_{d}^{t+1}=\left[\mu_{d}^{t}-x_{2}^{t}\left(\sum_{e \in Z_{d}} \widetilde{g}_{d, e}^{t}-G_{d}^{\min }\right)\right]^{+} \\
& u^{t+1}=\left[u^{t}-x_{3}^{t}\left(M^{t h}-\sum_{d=1}^{D} \sum_{e \in Z_{d}} I_{d, e}^{t}\left(\widehat{N}_{d, e}+Q^{-1}(1-z) u_{d, e}\right)\right)\right]^{+}
\end{aligned}
$$

Where, the iteration number is given by $t$, the $x_{1}, x_{2}$ and $x_{3}$ shows the small step sizes, whenever these step size are appropriately small the "Lagrange multipliers" can congregate to equilibrium points [30]. In the implementation process, initially maximum iteration count is given by $T_{\max }$, and initialization of $t=0, E>0 D>0$; the Lagrange multipliers are initializes as $0<\mu_{d}(0), 0<\lambda_{d}(0)$ and $0<u(0)$, though the threshold value of outage probability is $z \in[0,1], \mathfrak{s}_{d} \in[0,1]$. The upper channel estimation bound error in femto user link is $\left(\mathfrak{w}_{d, e} \in[0,1]\right)$ and the estimated error variance in femto user to macro user is $\left(v_{m, k} \in[0,1]\right)$. The maximal transmit power $\left(p_{m}^{\max }\right)$ and initialize power $\left(I_{d, e}\right)$ is set to greater than zero with same values at all subcarriers, also with the $M^{t h}$ interference. Afterwards the iteration is start from one, also for each $d$ and $e$ value it computes the transmit power $\left(I_{d, e}^{*}\right), h_{d, e}^{*}, \hat{\beta}_{d, e}$ and the updated Lagrange multipliers, till that the iteration has not finished.

\section{RESULT ANALYSIS}

The femto-cell can increase the spectrum reuse in order to provide the high spectral efficiency and the network capacity. Here, our aim is on association of users such as selection of BS, mode selection and channel allocation in order to optimize the power control at uplink communication in between the BSs and 
FCUs. The outage probability condition in a HetNet is applied in order to decrease the overall energy consumption, also this guarantee to all femto-cell users a bound of min and max for the worst level of outage probability. In consider HetNet environment, the FCUs and MCUs makes the request, based upon their requirement the channel and traffic details are acquired, then these things forwarded to our proposed RPRA for further optimization process to get maximum user throughput and optimize power transmission.

\subsection{Parameter initialization}

In order to simulate the results in Matlab2016b, we have used system configuration such as; $8 \mathrm{~Gb}$ RAM, i5 Intel Processor, 1Tb Hard disk and Windows 10 OS. The several considered necessary parameters that used in traffic condition scenarios is presented in table1. The growth of mobile users causes extra signal interference at FCUs and MCUs in small cells HetNet scenario; therefore, the femto-cell input is taken from real-time scenario like as video data [31] and audio data [32] in order to get realistic multi-media transmission. Under this considered traffic condition, we are going to take five scenarios such as; Scenario-A, Scenario-B, Scenario-C, Scenario-D and Scenario-E. In each of the scenario we have taken 1 macro-cell, 30 number of MCUs, 120 number of FCUs, where the location of femto-cell, FCUs and MCUs are generated randomly.

Table 1. Used parameter initialization

\begin{tabular}{ll}
\hline \multicolumn{1}{c}{ Parameter } & \multicolumn{1}{c}{ Value } \\
\hline Carrier Bandwidth & $2000 \mathrm{Mhz}$ \\
Subcarrier Bandwidth & $375 \mathrm{KHz}$ \\
System bandwidth & $10 \mathrm{MHz}$ \\
Maximum transmit power & $20 \mathrm{dBm}$ \\
Minimum transmit power & $0 \mathrm{dBm}$ \\
Antenna gain & $14 \mathrm{dBi}$ \\
Cell radius & $1 \mathrm{Km}$ \\
Correlation distance & $40 \mathrm{~m}$ \\
BS transmit power & $43 \mathrm{dBm}$ \\
User speed & $3 \mathrm{Km} / \mathrm{h}$ \\
Number of Macro-cell & 1 \\
Number of FCU & 30 \\
Number of MCU & 120 \\
\hline
\end{tabular}

\subsection{Performance analysis}

\subsubsection{Scenario-A}

In scenario-A, we have considered 1 macro-cell, 4 femto-cell, 30 number of MCUs and 120 number of FCUs. Here, Figure 2 shows the throughput in Mbps as per time interval, where our proposed model has performed significantly better at each time interval. Only in $110.9 \mathrm{~s}$ time interval there is a drop in our proposed approach, otherwise in taking average of all time interval our proposed model RPRA got 10.15 Mbps throughput, which is $13 \%$ more compare to HARQ-CC [33] and $25.5 \%$ more compare to HARQ-T1 [34]. Figure 3 shows the delay in s (second) as per the performed steps, where the average delay considering all the steps is $0.15 \mathrm{~s}$ from our RPRA model, $0.24 \mathrm{~s}$ obtained by HARQ-CC [33] and $2.92 \mathrm{~s}$ obtained by HARQ-T1 [34], therefore the delay in performance throughout the steps our model has consumed very less time compare to others.

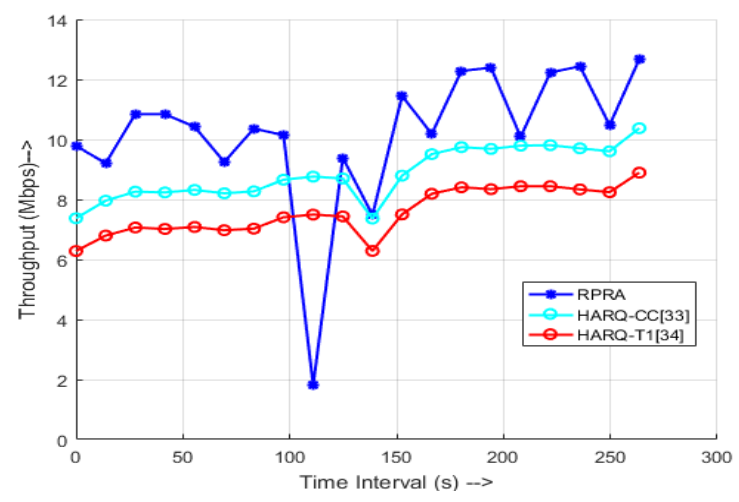

Figure 2. Throughput as per time interval

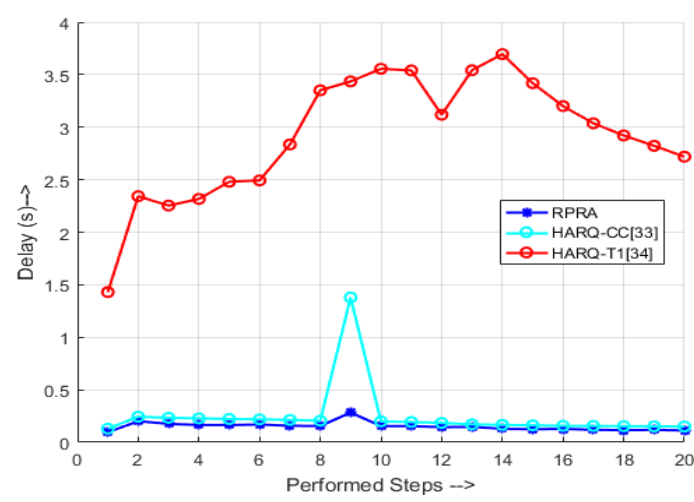

Figure 3. Delay as per the performed steps 


\subsubsection{Scenario-B}

In Scenario-B, we have considered 1 macro-cell, 8 femto-cell, 30 number of MCUs and 120 number of FCUs. Here, Figure 4 shows the throughput in Mbps as per time interval, where our proposed model RPRA got 21.95 Mbps average throughput, HARQ-CC has achieved 17.33 Mbps and HARQ-T1 has achieved 15.4 Mbps. Figure 5 shows the delay as per the performed steps, where the average delay considering all the steps is $1.14 \mathrm{~s}$ from our RPRA model, $0.52 \mathrm{~s}$ is obtained by HARQ-CC and $6.5 \mathrm{~s}$ is obtained by HARQ-T1.

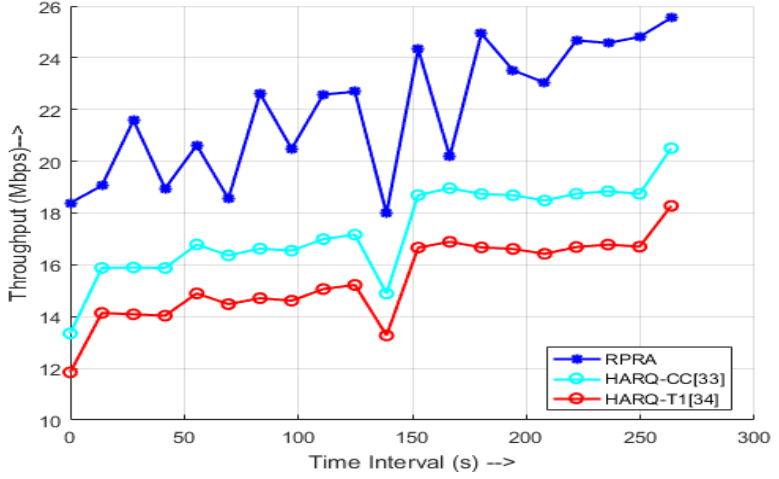

Figure 4. Throughput as per time interval

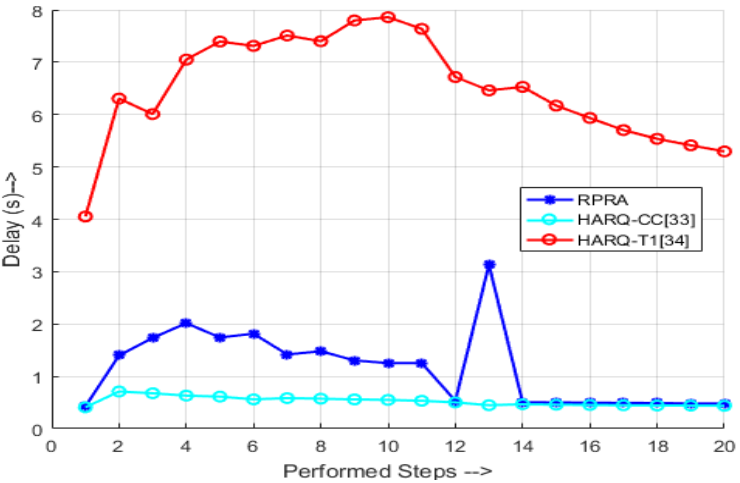

Figure 5. Delay as per the performed steps

\subsubsection{Scenario-C}

In Scenario-C, we have considered 1 macro-cell, 12 femto-cell, 30 number of MCUs and 120 number of FCUs. Figure 6 shows the throughput as per time interval, where dropout of throughput can be seen in $194 \mathrm{~s}, 249 \mathrm{~s}$ and $263 \mathrm{~s}$. However, still our proposed model average throughput of HetNet is $28 \mathrm{Mbps}$, which $16 \%$ more compare to HARQ-T1 and $5.8 \%$ more compare to HARQ-CC. Figure 7 shows the delay as per the performed steps, where the average delay considering all the steps is $0.77 \mathrm{~s}$ from our RPRA model, which $67 \%$ less compare to HARQ-T1 and $44 \%$ less compare to HARQ-CC.

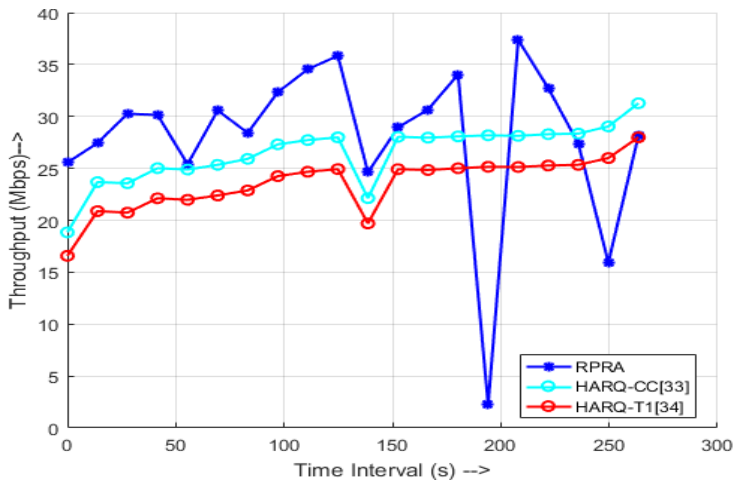

Figure 6. Throughput as per time interval

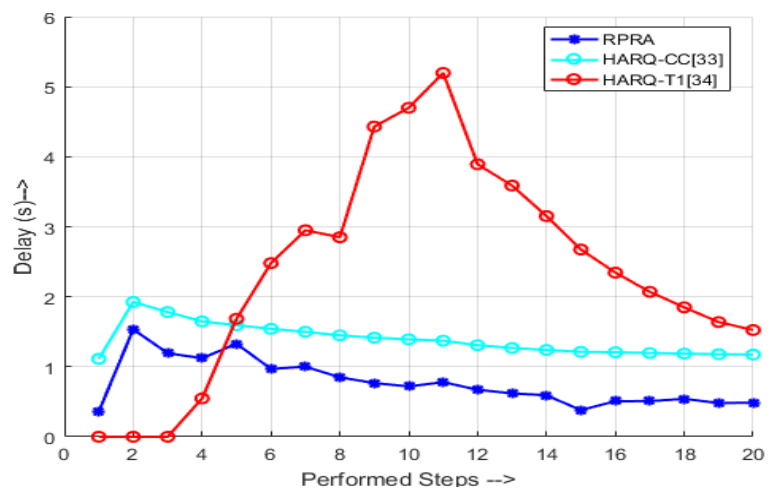

Figure 7. Delay as per the performed steps

\subsubsection{Scenario-D}

In Scenario-D, we have considered 1 macro-cell, 16 femto-cell, 30 number of MCUs and 120 number of FCUs. Figure 8 shows the throughput as per time interval, where proposed model average throughput of HetNet is $38 \mathrm{Mbps}$, which $17 \%$ more compare to HARQ-T1 and $8 \%$ more compare to HARQ-CC. Figure 9 shows the delay as per the performed steps, where the average delay considering all the steps is $1.6 \mathrm{~s}$ from our RPRA model, $2.16 \mathrm{~s}$ is obtained by HARQ-CC and $20 \mathrm{~s}$ is obtained by HARQ-T1. 


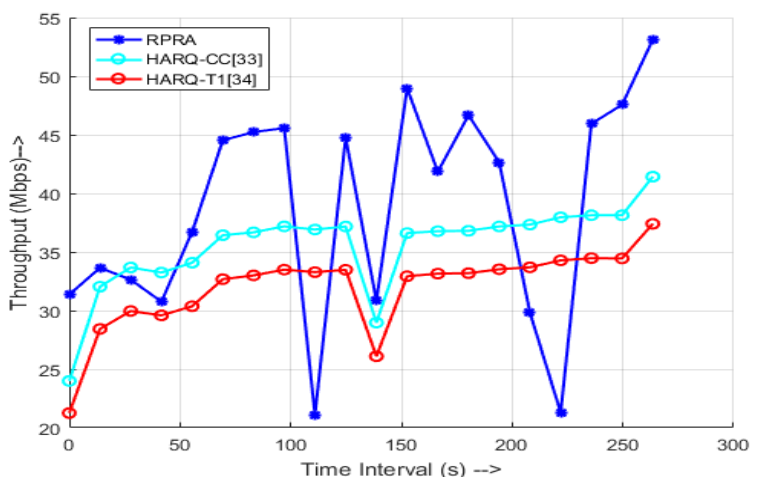

Figure 8. Throughput as per time interval

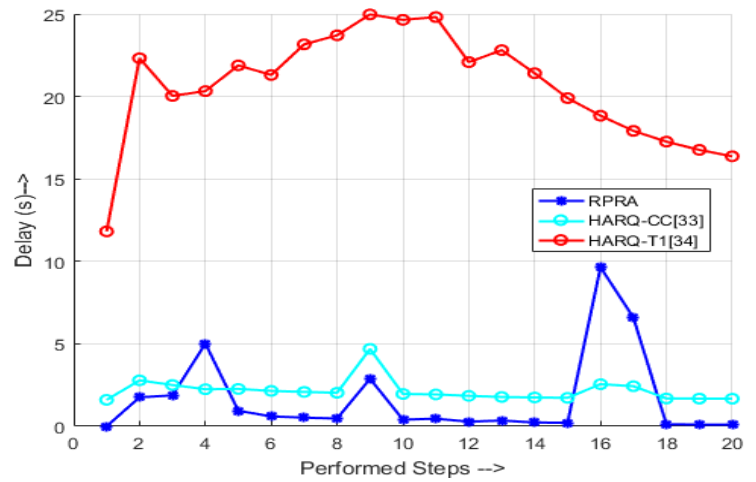

Figure 9. Delay as per the performed steps

\subsubsection{Scenario-E}

In Scenario-E, we have considered 1 macro-cell, 20 femto-cell, 30 number of MCUs and 120 number of FCUs. Figure 10 shows the throughput as per time interval, where proposed model average throughput of HetNet is $47 \mathrm{Mbps}, 43 \mathrm{Mbps}$ is obtained by HARQ-CC and $39.6 \mathrm{Mbps}$ is obtained by HARQ-T1. Figure 11 shows the delay as per the performed steps, where the average delay considering all the steps is $3.6 \mathrm{~s}$ from our RPRA model, $4.7 \mathrm{~s}$ is obtained by HARQ-CC and $13 \mathrm{~s}$ is obtained by HARQ-T1.

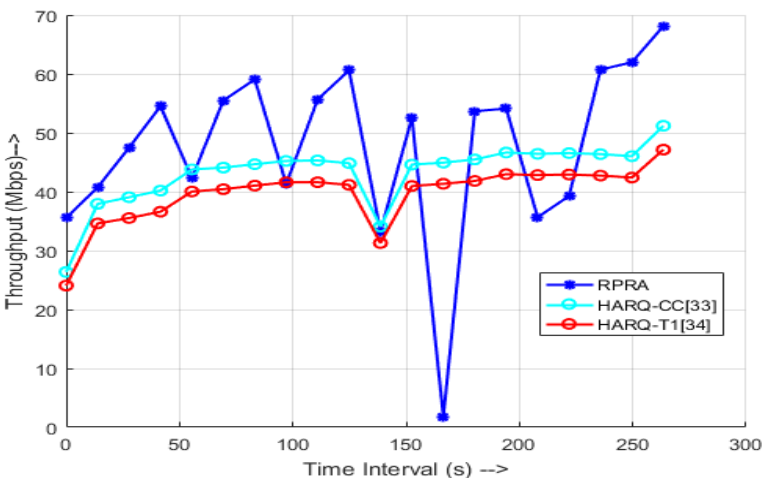

Figure 10. Throughput as per time interval

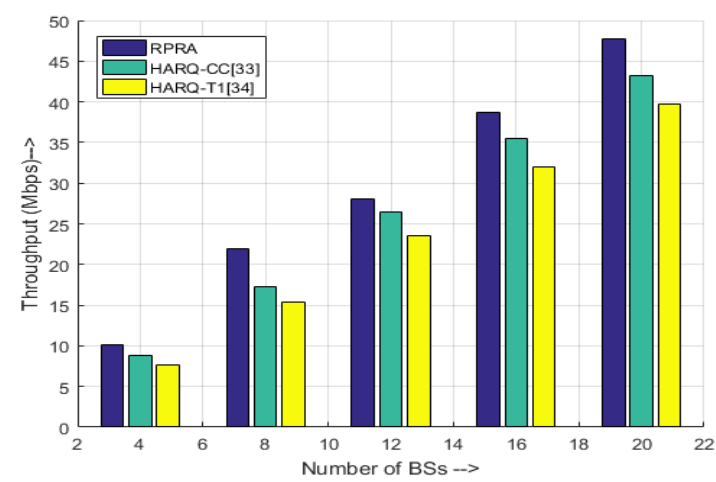

Figure 12. Average power transmission

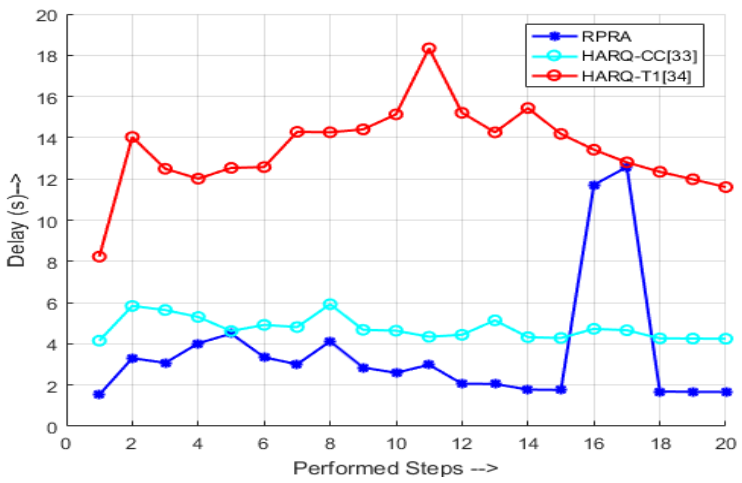

Figure 11. Delay as per the performed steps

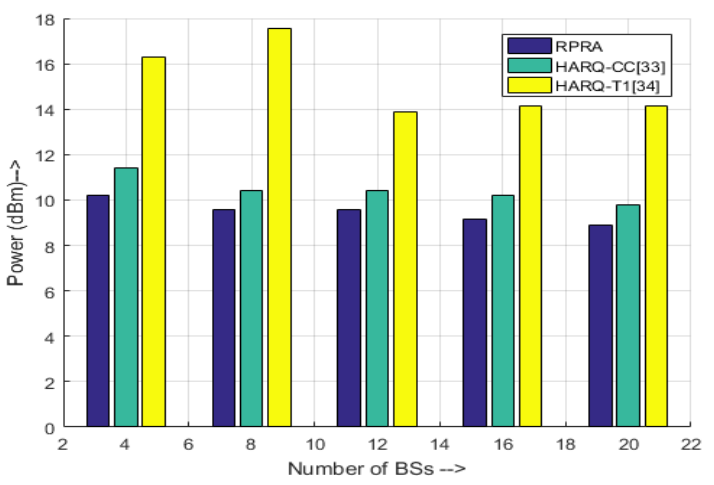

Figure 13. Obtained average throughput 


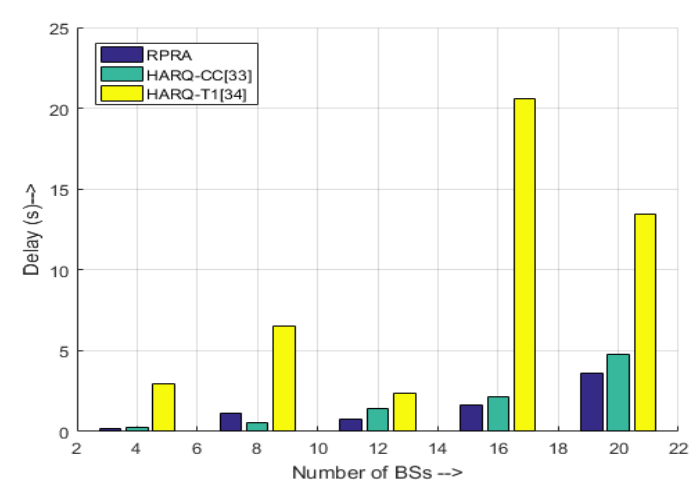

Figure 14. Average computational delay

Afterwards, as per the number of femto cell BSs in HetNet, we have computed the power transmission as per the BSs that shown in Figure 12. At 4 BSs, our proposed model has $10.22 \mathrm{dBm}$ average transmission power that is $10 \%$ less with respect to HARQ-CC and 37\% less with respect to HARQ-T1. However, in $20 \mathrm{BSs}$, RPRA has $18.9 \mathrm{dBm}$ average transmission power that is $8.8 \%$ less with respect to HARQ-CC and $36.9 \%$ less with respect to HARQ-T1. Figure 13 shows for the throughput as per number of BSs, and we can say in increment of BSs the throughput is also increasing, at $20 \mathrm{BSs}$ our proposed model has got $9 \%$ increment with respect to HARQ-CC and 16\% increment with respect to HARQ-T1. Figure 14 shows for the average computational delay and as per the analysis we got 73\% less delay w.r.t HARQ-T1 and $24 \%$ less w.r.t HARQ-CC at 20 number of BSs.

\section{CONCLUSION}

In this study, we consider one macro-cell and several number of femto-cells, where femto-cells has able to decrease the interference level with various channel signals that will help to optimize the spectral efficiency as per the covered cell area. The several number of femto-cells can be located in a macro-cell, therefore the BSs of femto-cells are located at much shorted distance to each other, which help to cover the poor transmission area present in a macro-cell. Moreover, the equipment becomes more energy efficient due to the shorted distance via decrement in transmission power that help to increase the life of battery. The proposed RPRA comprises two robust approach such as RPC and the RCAA, which can improve the spectral efficiency and user experiences at lower network coverage areas via eliminating the week coverage zones. Also provide the high user rate connection by effective interference in an efficient spectrum, lowering in transmission power and cost-effectiveness via less time delay. As per the obtained over all result analysis, we got $9 \%$ less power transmission w.r.t HARQ-CC and $37 \%$ less w.r.t HARQ-T1, in addition we got enhanced throughput rate in every scenario, therefore this validate the performance of our proposed model.

\section{REFERENCES}

[1] J. Andrews, H. Claussen, M. Dohler, S. Rangan, and M. Reed, "Femtocells: Past, present, and future," IEEE Journal on Selected Areas in Communications, vol. 30, no. 3, pp. 497-508, Apr 2012.

[2] D. Sun, X. Zhu, Z. Zeng, and S. Wan, "Downlink power control in cognitive femtocell networks," in 2011 International Conference on Wireless Communications and Signal Processing (WCSP), pp. 1-5, Nov 2011.

[3] C. Chen, F. Baccelli, L. Roullet, "Joint optimization of radio resources in small and macro cell networks," in Proc.IEEEVTCSpring, 2011.

[4] Q. Li,R. Hu,G. Wu, and Y. Qian, "Ontheoptimalmobile association in heterogeneous wireless relay networks," in Proc. IEEE INFOCOM, 2012.

[5] D. Fooladivanda and C. Rosenberg, "Joint resource allocation and user association for heterogeneous wireless cellular networks," IEEE Trans.Wireless Commun, vol. 12, no. 1, pp. 248-257, Jan 2013.

[6] L. Zhang, L. Yang, and T. Yang, "Cognitive interference management for LTE-A femtocells with distributed carrier selection," in 2010 IEEE 72nd Vehicular Technology Conference Fall (VTC 2010-Fall), pp. 1-5, Sept 2010.

[7] R. Xie, F. Yu, and H. Ji, "Spectrum sharing and resource allocation for energy-efficient heterogeneous cognitive radio networks with femtocells," in 2012 IEEE International Conference on Communications (ICC), pp. 1661-1665, Jun 2012

[8] S. Lien, Y. Lin, and K. Chen, "Cognitive and game-theoretical radio resource management for autonomous femtocells with QoS guarantees," IEEE Transactions on Wireless Communications, vol. 10, no. 7, pp. 2196-2206, Jul 2011. 
[9] D. López-Pérez, X. Chu, A. V. Vasilakos, and H. Claussen, "Power minimization based resource allocation for interference mitigation in OFDMA femtocell networks," IEEE Journal on Selected Areas in Communications, vol. 32, no. 2, pp. 333-344, 2014.

[10] H. ElSawy and E. Hossain, "Two-Tier HetNets with Cognitive Femtocells: Downlink Performance Modeling and Analysis in a Multichannel Environment," in IEEE Transactions on Mobile Computing, vol. 13, no. 3, pp. 649-663, Mar 2014.

[11] F. Rezaei, M. Hempel and H. Sharif, "LTE PHY performance analysis under 3GPP standards parameters," 2011 IEEE 16th International Workshop on Computer Aided Modeling and Design of Communication Links and Networks (CAMAD), Kyoto, pp. 102-106, 2011.

[12] G. Mansfield, "Femtocells in the US market-business drivers and consumer propositions," in Proc. 2008 FemtoCells Europe, pp. 1927- 1948.

[13] V. Chandrasekhar and J. G. Andrews, "Femtocell networks: a survey," IEEE Commun. Mag., vol. 46, no. 9, pp. 59-67, Sep. 2008.

[14] S. Yeh, S. Talwar, S. Lee, and H. Kim, "WiMAX femtocells: a perspective on network architecture, capacity, and coverage," IEEE Commun. Mag., vol. 46, no. 10, pp. 58-65, Oct. 2008.

[15] V. Chandrasekhar and J. Andrews, "Spectrum allocation in tiered cellular networks," IEEE Commun. Mag., vol. 57, no. 10, pp. 3059-3068, Oct. 2009.

[16] D. Lopez-Perez, A. Valcarce, and G. de la Roche, "OFDMA femtocells: a roadmap on interference avoidance," IEEE Commun. Mag., vol. 47, no. 9, pp. 41-48, Oct 2009.

[17] M. Neruda, J.Vrana, and R. Bestak, "Femtocells in 3G mobile networks," in Proc. 2009 IEEE Int. Conf. Syst., Signal Image Process., pp. 1-4, 2009.

[18] Q. Su, A. Huango, Z. Wu, G. Yu, Z. Zhang, K. Xu, and J. Yang, "A distributed dynamic spectrum access and power allocation algorithm for femtocell networks," in Proc. 2009 IEEE Int. Conf. Wireless Commun. Signal Process, 2009., pp. 1-5.

[19] C. Chen and S. J. Baek, "Multicast Scheduling for Relay-Based Heterogeneous Networks Using Rateless Codes," in IEEE Transactions on Mobile Computing, vol. 16, no. 11, pp. 3142-3155, Nov 2017.

[20] X. Ge, X. Li, H. Jin, J. Cheng and V. C. M. Leung, "Joint User Association and Scheduling for Load Balancing in Heterogeneous Networks," 2016 IEEE Global Communications Conference (GLOBECOM), 2016, pp. 1-6.

[21] H. Zhang, F. Fang, J. Cheng, K. Long, W. Wang and V. C. M. Leung, "Energy-Efficient Resource Allocation in NOMA Heterogeneous Networks," in IEEE Wireless Communications, vol. 25, no. 2, pp. 48-53, Apr 2018.

[22] F. H. Panahi, F. H. Panahi, G. Hattab, T. Ohtsuki and D. Cabrici, "Green heterogeneous networks via an intelligent power control strategy and D2D communications," 2017 IEEE 28th Annual International Symposium on Personal, Indoor, and Mobile Radio Communications (PIMRC), Montreal, QC, pp. 1-8, 2017.

[23] M. Wang, H. Gao and T. Lv, "Energy-Efficient User Association and Power Control in the Heterogeneous Network," in IEEE Access, vol. 5, pp. 5059-5068, 2017.

[24] M. S. Jin, S. A. Chae and D. I. Kim, "Per Cluster Based Opportunistic Power Control for Heterogeneous Networks," 2011 IEEE 73rd Vehicular Technology Conference (VTC Spring), Yokohama, 2011, pp. 1-5.

[25] C. W. Tan, "Optimal power control in Rayleigh-fading heterogeneous networks," 2011 Proceedings IEEE INFOCOM, Shanghai, 2011, pp. 2552-2560.

[26] Olivier Rioul and José Carlos Magossi, “On Shannon's Formula and Hartley's Rule: Beyond the Mathematical Coincidence" Entropy, vol. 16, no. 9, pp. 4892-4910, 2014.

[27] Arani A.H.; Mehbodniya A.; Omidi M.J.; Adachi F.; Saad W. Guven, I., "Distributed learning for energy efficient resource management in selforganizing heterogeneous networks," IEEE Trans. Veh. Technol., vol. 66, pp. 9287-9303, 2017.

[28] Zhang, H. J., Liu H., Cheng J., Leung V. C. M., "Downlink energy efficiency of power allocation and wireless backhaul bandwidth allocation in heterogeneous small cell networks," IEEE Trans. Commun, vol. 95, pp. 1-13, 2017.

[29] K. C. Almeida and R. Salgado, "Optimal power flow solutions under variable load conditions," in IEEE Transactions on Power Systems, vol. 15, no. 4, pp. 1204-1211, Nov 2000.

[30] Horst R., Pardalos P. M., Thoai N.V., Introduction to Global Optimization, 2nd ed, USA: Kluwer Academic Publishers, 2000.

[31] P. Seeling and M. Reisslein, "Video Transport Evaluation With H.264 Video Traces," in IEEE Communications Surveys \& Tutorials, Fourth Quarter 2012, vol. 14, no. 4, pp. 1142-1165, 2012.

[32] Christos Bouras, Vasileios Kokkinos and Andreas Papazois, "A simulation framework for lte-a systems with femtocell overlays," ACM New York, NY, USA, 2012.

[33] M. Sheng, J. Wen, J. Li, B. Liang and X. Wang, "Performance Analysis of Heterogeneous Cellular Networks with HARQ Under Correlated Interference," in IEEE Transactions on Wireless Communications, vol. 16, no. 12 , pp. 8377-8389, Dec 2017.

[34] Evolved Universal Terrestrial Radio Access (E-UTRA); Radio Resource Control (RRC); Protocol Specification, document TS 36.331, Rev. 12.5.0, 3GPP, Mar 2015. 